\title{
ELECTRE METHODS: MAIN FEATURES AND RECENT DEVELOPMENTS
}

\author{
Jose Rui Figueira \\ Center for Management Studies, Technical \\ University of Lisbon \\ Tagus Park, Av. Cavaco Silva, 2780-990 Porto Salvo, Portugal \\ Phone: ++ 351214233 507; E-mail: figueira@ist.utl.pt
}

Key words: $\quad M C D A$, Electre methods, choosing, ranking, sorting

\section{EXTENDED ABSTRACT}

We present main characteristics of Electre family methods, designed for multiple criteria decision aiding. These methods use as a preference model an outranking relation in the set of actions - it is constructed in result of concordance and non-discordance tests involving a specific input preference information. After a brief description of the constructivist conception in which the Electre methods are inserted, we present the main features of these methods. We discuss such characteristic features as: the possibility of taking into account positive and negative reasons in the modeling of preferences, without any need for recoding the data; using of thresholds for taking into account the imperfect knowledge of data; the absence of systematic compensation between "gains" and "losses". The main weaknesses are also presented. Then, some aspects related to new developments are outlined. These are related to some new methodological developments, new procedures, axiomatic analysis, software tools, and several other aspects. The paper ends with conclusions. 